Kairos. Journal of Philosophy \& Science 20, 2018

Center for the Philosophy of Sciences of Lisbon University

\title{
The Conventionality of Simultaneity and Einstein's Conventionality of Geometry
}

\author{
Mário Bacelar Valente \\ Universidad Pablo de Olavide (Spain) \\ mar.bacelar@gmail.com
}

\begin{abstract}
The conventionality of simultaneity thesis as established by Reichenbach and Grünbaum is related to the partial freedom in the definition of simultaneity in an inertial reference frame. An apparently altogether different issue is that of the conventionality of spatial geometry, or more generally the conventionality of chronogeometry when taking also into account the conventionality of the uniformity of time. Here we will consider Einstein's version of the conventionality of (chrono) geometry, according to which we might adopt a different spatial geometry and a particular definition of equality of successive time intervals. The choice of a particular chronogeometry would not imply any change in a theory, since its "physical part" can be changed in a way that, regarding experimental results, the theory is the same. Here, we will make the case that the conventionality of simultaneity is closely related to Einstein's conventionality of chronogeometry, as another conventional element leading to it.
\end{abstract}

DOI 10.2478/kjps-2018-0008

\section{Introduction}

Historically, the thesis of the conventionality of simultaneity emerged from Reichenbach's work on the issue of the definition of simultaneity, which was initially based on Einstein's remarks about the definition of simultaneity in an inertial reference frame. Two apparently unconnected issues are that of the conventionality of spatial geometry and the related issue of the conventionality of the uniformity of time. Einstein developed 
what we might consider his version of the conventionality of geometry thesis, which, by also taking into account the conventionality of the uniformity of time, he applied to the space-time geometry (which we will refer to as chronogeometry).

In section 2, we review Einstein's views on the definition of simultaneity and the early work on the conventionality of simultaneity made by Reichenbach (1924, 1927), Grünbaum (1955, 1968), Edwards (1963), Winnie (1970), and Giannoni (1978). In section 3, we review Einstein's thesis of the conventionality of chronogeometry. Finally, in section 4 , we will make the case that the conventionality of simultaneity is one of the elements that gives rise to Einstein's conventionality of chronogeometry, which originally did not include the definition of simultaneity as one of its conventional elements. An implicit aspect of this view is that it can be made by just taking into account the above mentioned early works on the thesis of the conventionality of simultaneity; we do not make any attempt at this point to relate this view to important approaches to the conventionality of simultaneity developed afterwards. ${ }^{1}$

\section{The Thesis of the Conventionality of Simultaneity}

With the special theory of relativity, the definition of (coordinate) time of an inertial reference frame became dependent on the stipulation of distant simultaneity. To say that the time at a point $A$ and the time at a point $B$ are the same presupposes the establishment of a relation between the time at $A$ and the time at $B$, which enables us to reach this conclusion. Einstein defines distant simultaneity by a particular stipulation of synchrony of spatially separated clocks. According to Einstein:

"It is not possible to compare the time of an event at A with one at $B$ without a further stipulation ... the latter can now be determined by establishing by definition that the "time" needed for the light to travel from A to $B$ is equal to the "time" it needs to travel from $B$ to $A$. For, suppose a ray of light leaves from A toward B at "A-time" $t_{A}$, is reflect-

See, e.g., Janis, 2014; Valente 2018. 
ed from B toward A at "B-time" $t_{B}$, and arrives back at $A$ at "A-time" $t_{A}$ '. The two clocks are synchronous by definition if $t_{B}-t_{A}=t_{A}^{\prime}-t_{B}{ }^{\prime \prime}$

The definition of coordinate time is made by stipulating that the oneway speed of light from $A$ to $B$ is the same as the one-way speed of light from $B$ to $A$. In a later work Einstein remarks that we can measure the speed of light in a given direction "only if the definition of [coordinate] time we are seeking has already been given". ${ }^{3}$ That is, previous to the definition of coordinate time we cannot determine the speed of light (in fact, there is not even a meaningful notion of velocity). Accordingly, Einstein presupposes the identity of the one-way speed of light in opposite directions to enable the establishment of the coordinate time of an inertial reference frame: "we shall now stipulate that the velocity of the propagation of light in vacuum from some point $A$ to some point $B$ is the same as that from $B$ to $A " .{ }^{4}$ Einstein returns to this issue in 1917, mentioning that we are facing what he calls a logical circle. The definition of simultaneity rests on taking the speed of light from $A$ to $B$ to be the same as that from $B$ to $A$, "but an examination of this supposition would only be possible if we already had at our disposal the means of measuring time. It would thus appear as though we were moving here in a logical circle". ${ }^{5}$ This is why Einstein speaks of stipulation when referring to the one-way speed of light:

"That light requires the same time to traverse the path $[A B]$ as for the path $[B A]$ is in reality neither a supposition

\footnotetext{
2 Einstein, 1905, 142.

3 Einstein, $1911,345$.

4 Einstein, 1911, 345. There seems to be some inconsistency here, since, as mentioned, previous to the definition of coordinate time there is no meaningful notion of velocity. But this seems to be more a case of imprecision in the wording. We might speak of a symmetrical physical process of propagation from $A$ to $B$ and from $B$ to $A$, instead of the one-way speed of light. In a work from 1910, Einstein, basically, does this: "we have no reason to believe that the phenomena of signal transmission in the direction $A B$ will differ in any way whatsoever from the phenomena of signal transmission in the direction BA. In this case there is, obviously, only one way of regulating the clock at B against the clock at $A$ in such a manner that the signal traveling from $A$ to $B$ would take the same amount of time ... as the signal from $B$ to A" (Einstein, 1910, 126).
}

5 Einstein, 1917, 272. 
nor a hypothesis about the physical nature of light, but a stipulation which I can make of my own freewill in order to arrive at a definition of simultaneity" ${ }^{\prime \prime}$.

Reichenbach was influenced by Einstein's writings on the definition of simultaneity, leading him to work on this subject:

"Einstein suggested that the definition of synchronous time at every point of a chosen coordinate system necessitates a physical process spreading with a certain velocity and permitting a comparison of clocks at different points. Subsequently a hypothesis must be formulated about the state of motion of this process relative to the coordinate system. The time of the coordinate system and the simultaneity at distant points depend on this hypothesis. Yet it is impossible to determine this state of motion; such a determination presupposes a time definition ... there is, therefore, a certain arbitrariness contained in any "coordinate time". ${ }^{7}$

Exploring the circularity existing between the determination of the oneway speed of light and the determination of simultaneity - to determine one we need to know the other -, Reichenbach considered, besides Einstein's standard definition, other alternative definitions of simultaneity. According to Reichenbach:

"[A definition of simultaneity] according to the formula $t_{2}=\left(t_{1}+t_{2}\right) / 2$ is not false because such a definition is arbitrary ... the inconvenience of such a notion of [coordinate] time resides in the fact that it would violate the principle of causality." 8

$6 \quad$ Einstein, 1917, 272.

7 Reichenbach, 1920, 9-10.

8 Reichenbach, 1922, 115. In this expression $t_{1}$ is the time at which a light signal is emitted at one point, and $t_{2}$ is the time when it arrives at another point. 
In fact, the related ideas of causal chain and first signal, resulting from the existence of a limiting velocity in the theory, led Reichenbach to the so-called $\varepsilon$-definition of simultaneity. ${ }^{9}$ Einstein's definition of simultaneity (or, equivalently, Einstein's definition of coordinate time) corresponds to adopting the rule $t_{B}=t_{A}+1 / 2\left(t_{A}{ }^{\prime}-t_{A}\right)$. By imposing just the time order $t_{A}{ }^{\prime}>t_{B}>t_{A}$, the previous expression can be generalized to $\mathrm{t}_{\mathrm{B}}=\mathrm{t}_{\mathrm{A}}+\varepsilon\left(\mathrm{t}_{\mathrm{A}}{ }^{\prime}-\mathrm{t}_{\mathrm{A}}\right)$, with $0<\varepsilon<1$. According to Reichenbach, any value of $\varepsilon$ between 0 and 1 provides an equally valid definition of simultaneity.

Reichenbach's views on the conventionality of simultaneity were taken up by Grünbaum (1955) who further developed the subject. In particular, Grünbaum showed that we can adopt in two inertial reference frames (in relative motion) different conventions of simultaneity so that two events that are taken to be simultaneous in one of the inertial reference frames are also simultaneous in the other, i.e. there is no relativity of simultaneity. To arrive at this result we simply need to stipulate appropriate values for the one-way speed of light in two opposite directions in each frame, corresponding to particular values of $\varepsilon$ in each inertial reference frame. ${ }^{10}$ According to Grünbaum:

"The conventionality of simultaneity allows but does not entail our choosing the same value $\varepsilon=1 / 2$ for all directions within every system. In each system this choice assures the equality of the one-way velocities of light in opposite directions by yielding equal one-way transit times ... for equal distances. The ratio of these one-way transit times is $\varepsilon /(1-\varepsilon)$, and therefore, in the case of $\varepsilon \neq 1 / 2$, these one-way times are unequal. But ... no fact of nature ... would be contradicted if we choose values of $\varepsilon \neq 1 / 2$ for each inertial system, thereby making the velocity of light different from $\mathrm{c}$ in both senses along each direction in all inertial systems." 11

After Grünbaum brought attention to Reichenbach's work on the conventionality of simultaneity there has been ongoing work on the sub-

9 Reichenbach, 1924, 31-40; see also Rynasiewicz, 2003.

10 Grünbaum, 1955, 455-6.

11 Grünbaum, 1968, 308. 
ject. ${ }^{12}$ For our purpose we will only need to take into account some of the early works made after Grünbaum's 1955 essay.

In 1963, Edwards extended Reichenbach and Grünbaum's works on the conventionality of simultaneity by showing that the Lorentz transformations between inertial reference frames can be generalized in a way that corresponds to the adoption of any permissible values of $\varepsilon$ and $\varepsilon$ ' for each inertial frame. ${ }^{13}$ According to Edwards, the generalized Lorentz transformations are equivalent to those standardly adopted (for which $\varepsilon=\varepsilon^{\prime}=1 / 2$ ); in particular, they all agree with the experimental results. This is due to the fact that mathematical expressions that are different when adopting a different one-way speed of light, one example being the time dilation formula, do not correspond to what Edwards refers to as quantities that are directly observable. ${ }^{14}$

Edwards' work was further developed by Winnie (1970) that showed that the mathematical expressions corresponding to several kinematical effects predicted by the theory had conventional elements due to the choice of the one-way speed of light. Specifically, Winnie obtained synchrony-dependent formulas for the relative speed, the length contraction, and the time dilation. However, Winnie also showed that the mathematical expressions corresponding to observable length contraction and time dilation effects were synchrony-independent, i.e. the formulas were the same independently of the adopted $\varepsilon$. Adopting an approach different from Edwards', Winnie deduced the generalized Lorentz transformations between two inertial reference frames with epsilon values $\varepsilon$ and $\varepsilon$ ' (which he called $\varepsilon$-Lorentz transformations). Winnie concluded that "different choices of $\varepsilon$ results in kinematically equivalent versions of the special theory [of relativity]". ${ }^{15}$

12 See, e.g., Jammer, 2006; Janis, 2014.

13 Edwards, 1963, 486; see also Brown, 2005, 102. Edwards made his point by considering causality limitations on the one-way speed of light leading to the expression $C / 2 \leq c(\alpha, \beta, \gamma) \leq \infty$, where $C$ is the two-way speed of light, and $c(\alpha, \beta, \gamma)$ is the one-way speed of light in the direction indicated by the direction cosines $\alpha, \beta$ and $\gamma$. This expression corresponds to having $0 \leq \varepsilon \leq 1$.

14 Edwards, 1963, 489; see also Jammer, 2006, 251.

15 Winnie, 1970, 237. 
We see that we can adopt an anisotropic one-way speed of light corresponding to a non-standard synchronization of clocks; the adoption of an $\varepsilon \neq 1 / 2$ or $\varepsilon^{\prime} \neq 1 / 2$ (or both) goes hand in hand with a generalization of the Lorentz transformations and a formulation of kinematical relativity compatible with this generalization. At this point we might ask about relativistic dynamics in the context of the adoption of the generalized Lorentz transformations. Also, since in the generalized case we have an anisotropic one-way speed of light, what to make of electrodynamics, which is formulated with an isotropic speed of light? These and other issues were addressed by Giannoni (1978).

Giannoni developed a generalization of relativistic dynamics for the case of a non-standard synchronization (or, equivalently, the case of an anisotropic one-way speed of light). Like in the case of relativistic kinematics we find that there are concepts whose corresponding mathematical expressions depend on the adopted definition of simultaneity. For example, "inertial mass is a synchronization dependent as well as velocity dependent quantity, and it is as conventional as synchronization is conventional". ${ }^{16}$ Electrodynamics can also be extended to the case of an anisotropic one-way speed of light. Adopting an $\varepsilon \neq 1 / 2$ in an inertial reference frame, the one-way speed of light along the positive direction of the $x$-axis is $c / 2 \varepsilon$ and the one-way speed of light along the negative direction of the $x$-axis is $c / 2(1-\varepsilon) .{ }^{17}$ Consistently with this assumption, the corresponding generalized electrodynamics predicts a plane wave traveling in the positive $x$ direction with a speed of $c / 2 \varepsilon$ and a plane wave traveling in the negative $x$ direction with a speed of $c / 2(1-\varepsilon) .{ }^{18}$ The extension of the work of Reichenbach and Grünbaum made by Edwards, Winnie, and Giannoni, shows that the conventionality of simultaneity thesis does not lead to any contradiction when considering the relevant physical theories as a whole. ${ }^{19}$

\footnotetext{
16 Giannoni, 1978, 29.

17 See, e.g., Winnie, 1970, 83.

18 Giannoni, 1978, 37-8.

19 We must notice that the conventionality of simultaneity thesis is not uncontested in the philosophy of relativity (see, e.g. Selleri, 1996; Anderson et al., 1998; Janis, 2014; Valente 2017a). It is beyond the scope of this work to address this issue here.
} 


\section{The Conventionality of Geometry According to Einstein}

Einstein's mature views on geometry were presented in his well-known "Geometry and experience", from 1921. Here, Einstein fully articulated ideas that he had been writing about at least since $1914 .{ }^{20}$ Einstein makes a distinction between axiomatic geometry and practical geometry. On its own, axiomatic geometry has a "merely logical-formal character", ${ }^{21}$ i.e. it does not apply directly to nature. For this purpose, it is necessary to complete the axioms of geometry by coordinating the "empty conceptual schemata", 22 formed by undefined words like "point" and "straight line", to the "real objects of experience". ${ }^{23}$ For this Einstein relies on the notion of practically-rigid body. ${ }^{24}$ Einstein considers that:

"Solid bodies are related, with respect to their possible dispositions, as are bodies in Euclidean geometry of three dimensions. Then the propositions of Euclid contain affirmations as to the behavior of practically-rigid bodies." 25

A more precise presentation of practically-rigid bodies, making explicit that a practically-rigid body is taken not to have its length affected under transport, is made in Einstein's 1917 book:

"[We] supplement the propositions of Euclidean geometry by the single proposition that two points on a practically rigid body always correspond to the same distance (line-in-

$\overline{20}$ See, Einstein, 1914, 78; Einstein, 1916, 148; Einstein, 1917, 250-1; Einstein, 1920, 144-6.

21 Einstein, $1921 \mathrm{a}, 211$.

22 Einstein, $1921 \mathrm{a}, 234$.

23 Einstein, $1921 \mathrm{a}, 235$.

24 We can define a practically-rigid body by what Darrigol called "chrono-optical control of rigidity" (Darrigol, 2015, 167). According to Darrigol, "rigid rods can be constructed by clock-based optical control, exploiting the constancy of the [two-way] velocity of light" (Darrigol, 2015, 179). As Darrigol mentioned, this concept of (small) extended rigid body is not incompatible with relativity (special or general).

25 Einstein, $1921 \mathrm{a}, 235$. 
terval), independently of any changes in position to which we may subject the body." 26

By adopting the standpoint of practical geometry, "one grants reality to the practically rigid body". ${ }^{27}$ Accordingly:

"The concept of distance corresponds to something that can be experienced. Geometry then contains statements about possible experiments; it is a physical science directly subjected to experimental testing." 28

Einstein adopts, provisionally, practical geometry because regarding the notions of measuring-rod (i.e. solid body) and clock, "in the present stage of development of theoretical physics these concepts must still be employed as independent concepts". ${ }^{29}$ This is the reason that justifies the adoption of practical geometry but at the same time it shows its weakness, since the adoption of practical geometry results from an "incompleteness" of the theory at its present stage of development. In Einstein's words, the theory "does not yet exist at all as a finished product". ${ }^{30}$ In his autobiographical notes Einstein mentions that:

"One is struck [by the fact] that the theory (except for the four-dimensional space) introduces two kinds of physical things, i.e., (1) measuring rods and clocks, (2) all other things, e.g., the electromagnetic field, the material point, etc. This, in a certain sense, is inconsistent; strictly speaking measuring rods and clocks would have to be represented as solutions of the basic equations (objects consisting of moving atomic configurations), not as it were, as theoretically self-sufficient entities ... it was better to permit such

\footnotetext{
26 Einstein, 1917, 251.

27 Einstein, 1924, 326.

28 Einstein, 1924, 326.

29 Einstein, 1921 a, 213

30 Einstein, 1949b, 678.
} 
inconsistency - with the obligation, however, of eliminating it at a later stage of the theory." ${ }^{31}$

In this way rods and clocks are not treated as complex solutions of the theory's equations. They enter the theory as independent concepts that are directly coordinated with geometrical objects - spatial intervals and time intervals. ${ }^{32}$ Specifically, in practical geometry we make a direct coordination of a practically-rigid body with a mathematical spatial interval that corresponds to the length of the practically-rigid body. Since the practically-rigid body is taken to maintain its length under transport, it follows the equality (congruence) of the spatial intervals corresponding to the lengths of identical practically-rigid bodies (rods) located at different places. Let us now set ourselves in an imagined later stage of development of classical field theory in which rods and clocks are not anymore independent concepts and can be seen as solutions of field equations. ${ }^{33}$ Let us look again into the view that practically-rigid bodies maintain their length under transport, and that we identify distant spatial intervals with the (identical) length of practically-rigid bodies, in this way arriving at the equality (congruence) of the spatial intervals. From a conventionalist perspective, this is a stipulation since we can never know if a (real) material body (taken to be described by the theory) is actually rigid when located at a particular place or being moved around. While Einstein considers that, provisionally, we must adopt practical geometry, he still considers that sub specie aeterni the direct relation between the practically-rigid body and geometry is not well-grounded:

"Under closer inspection the real solid bodies in nature are not rigid, because their geometrical behavior, that is,

\footnotetext{
31 Einstein, 1949a, 59 and 61.

32 See, e.g., Einstein, 1922, 323.

33 We must bear in mind that Einstein, after finding his general relativistic field equations in late 1915, went on with his "research program" searching for a unified field theory (see, e.g., Goenner, 2004). For example, some weeks after the presentation of "Geometry and experience", Einstein explored a further development of general relativity taking into account Weyl's tentative theory unifying gravitation and electromagnetism (Einstein, $1921 \mathrm{~b}$ ). Einstein expected that at a later stage of development of the theory (in terms of a unified field theory) the inconsistency of treating rods and clocks as theoretically self-sufficient entities would be eliminated.
} 
their possibilities of relative disposition, depend upon temperature, external forces, etc." 34

In Einstein's view, we cannot rely on theory to take into account these factors, since a theory presupposes the adoption of a particular geometry. In this way, we never know if the bodies are actually rigid or not. This leads Einstein to advocate in principle, if not in practice, a conventionalist view on geometry:

"Geometry (G) predicates nothing about the behavior of real things, but only geometry together with the totality $(P)$ of physical laws can do so. Using symbols, we may say that only the sum of $(G)+(P)$ is subject to experimental verification. Thus $(G)$ may be chosen arbitrarily, and also parts of $(P)$; all these laws are conventions. All that is necessary to avoid contradictions is to choose the remainder of $(P)$ so that $(G)$ and the whole of $(P)$ are together in accord with experience." 35

In later years, Einstein seems to have maintained this, in principle, position. We can see this in the text known as "reply to criticism" where there

34 Einstein, $1921 \mathrm{a}, 212$. Einstein position on rigid bodies in "Geometry and experience" is nuanced. In his view: "it is not a difficult task to determine the physical state of a measuring-rod so accurately that its behavior relatively to other measuring-bodies shall be sufficiently free from ambiguity to allow it to be substituted for the "rigid" body. It is to measuring-bodies of this kind that statements as to rigid bodies must be referred" (Einstein, 1921a, 237). Einstein's dissatisfaction with the notion of rigid body and the reasons for it are clearer in his "Nobel lecture" in which Einstein mentions that: "In nature, the rigid body is only approximately realized and not even to an arbitrary degree of approximation. Thus, this concept does not strictly obey the "reality postulate". Moreover, it seems logically unjustified to base all physical considerations on the rigid, that is, the solid body and then, in the end, to reconstruct it atomistically again with the aid of the fundamental laws of physics that are, in turn, constructed with the aid of the concept of a rigid measuring-body" (Einstein, 1923, 75).

35 Einstein, 1921a, 212. Einstein presents this view as Poincaré's standpoint (see, e.g., Einstein, 1925, 217; Einstein, 1949b, 677). The family membership of Einstein's conventionalism is an intricate issue that will not be dealt with in this work. However, a brief remark seems appropriate at this point. The relation of Einstein's conventionality of geometry and Poincaré's might not be as direct as Einstein thought. There is, e.g., the view that Einstein developed his own conventionality of geometry, borrowing elements from Poincaré and others (Paty, 1992), there is also the view that Einstein articulated a geometrical version of Duhem's holism, not Poincaré's conventionalism (Grünbaum, 1976), or 
is an imaginary dialogue, between Poincaré (and at the end a non-positivist) and Reichenbach. In it Einstein's "Poincaré" mentioned:

"The empirically given bodies are not rigid, and consequently cannot be used for the embodiment of geometric intervals. Therefore, the theorems of geometry are not verifiable ... why should it consequently not be entirely up to me to choose geometry according to my own convenience (i.e. Euclidean) and to fit the remaining (in the usual sense "physical") laws to this choice in such a manner that there can arise no contradiction of the whole with experience? ${ }^{36}$

This dialogue seems to have the objective of defending a conventionalist/holistic view, ${ }^{37}$ even if in it, Einstein's "Reichenbach" endorsed a view compatible with Einstein's practical geometry. In fact, earlier in this text, as Howard (2010) noticed, Einstein endorsed a conventionalist/ holistic stance:

"The theoretical attitude here advocated is distinct from that of Kant only by the fact that we do not conceive of the "categories" as unalterable (conditioned by the nature of the understanding) but as (in the logical sense) free conventions." 38

For the purpose of this work we must also notice that Einstein's views on geometry are not restricted to the case of three-dimensional (spatial) geometry; according to him they include also Riemannian chronogeometry. ${ }^{39}$ While Einstein was not very explicit regarding what we might call the practical chronometry associated with clocks, practical chronometry is established, mutatis mutandis, as it is practical geometry. ${ }^{40}$ Implicitly,

the view that, after all, Einstein's interpretation is accurate (Ben-Menahem, 2006, 129-30, footnote 77). For the purpose of this work we do not need to address this issue.

36 Einstein, 1949b, 677.

37 For a similar interpretation see, e.g., Giovanelli, 2013, 3854-5.

38 Einstein, 1949b, 674.

39 Einstein, $1921 \mathrm{a}, 213$.

40 Einstein, 1921a, 213-4. 
Einstein takes the practical chronogeometry of Riemann to incorporate practical (spatial) geometry and practical chronometry. From a conventionalist position, the situation is the same with clocks as with rigid bodies (measuring rods). According to Einstein, like in the case of a putative rigid body, a clock that always has the same rate "does not find [its] exact correspondence in the real world". ${ }^{41}$ This means, from a conventionalist stance, that when we coordinate the reading of a clock (e.g. the motion of its hands) with a mathematical time interval, the equality (congruence) of successive time intervals is a convention. It is important to notice that Einstein only considers two conventional elements and their practical counterpart due to adopting rods and clocks as independent concepts (provisionally, at the present stage of the theory). In relation to (spatial) geometry we have the conventionality of the equality (congruence) of spatial intervals, and in relation to chronometry we have the conventionality of the uniformity of time (congruence of successive time intervals).

\section{The Conventionality of Simultaneity and the Conventionality of Chronogeometry}

As we have seen, Einstein's views on the definition of simultaneity influenced Reichenbach's work on what was later called the conventionality of simultaneity thesis. Reichenbach gave a detailed presentation of his views in his 1927 book. ${ }^{42}$ Einstein wrote a review of this book, emphasizing Reichenbach's treatment of this subject:

"Special care has been taken to ferret out clearly what in the relativistic definition of simultaneity is a logically arbitrary decree and what in it is a hypothesis, i.e. an assumption about the constitution of nature." 43

It is clear that Einstein knew about the $\varepsilon$-definition of simultaneity. However, it does not seem that Einstein ever considered the necessity of taking into account the conventionality of simultaneity in relation to

\footnotetext{
$41 \quad$ Einstein, 1921 a, 212.

42 Reichenbach, 1927, 123-147.

43 Cited in Jammer, 2006, 191.
} 
his views on the conventionality of chronogeometry. As mentioned, for Einstein the conventionality of chronogeometry was just related to the conventionality of the equality (congruence) of spatial intervals and the conventionality of the uniformity of time (congruence of successive time intervals). Reichenbach also considered them as unrelated issues. ${ }^{44} \mathrm{An}$ author that somewhat related the conventionality of simultaneity and the conventionality of chronogeometry was Grünbaum. He refers to the geochronometry ${ }^{45}$ of space-time, ${ }^{46}$ and writes that the "metrical comparison of separate spatial and temporal intervals required for geochronometry involve rigid rods and isochronous clocks". ${ }^{47}$ According to Grünbaum, "the rigidity or self-congruence of [a] standard under transport [is] decreed by convention, and similarly for the periodic devices which are held to be isochronous (uniform) clocks". ${ }^{48}$ This led Grünbaum to affirm the conventionality of the geochronometry. ${ }^{49}$ Grünbaum mentioned that "the epistemological status of the Riemman-Poincaré conventionality of congruence is fully analogous to that of Einstein's conventionality of simultaneity". ${ }^{50}$ This led him to combine the two theses under the name of "geochronometric conventionalism". ${ }^{51}$ However, Grünbaum was just referring to their "complete epistemological affinity". ${ }^{52}$

44 See, e.g., Reichenbach, 1927. In a remark Reichenbach mentions the three conventional elements (spatial congruence, uniformity of time, and simultaneity) together: "Certain assumptions about rigid bodies as a standard of measurement for distant spatial segments must be laid down before the question as to the geometry of real space takes a definite meaning. Hence the relativity of geometry ... To this idea of Helmholtz, Einstein added an analogous idea respecting time; simultaneity (and uniformity) can only be discussed upon the assumption of a certain extension of Helmholtzian conventionalism from space to time" (Reichenbach, 1928, 233). There is, however, no integrated analysis of these issues made by Reichenbach.

45 In this work, we have adopted the term "chronogeometry" instead of the term "geochronometry".

46 Grünbaum, 1962, 405.

47 Grünbaum, 1962, 408.

48 Grünbaum, 1962, 414.

49 Grünbaum, 1962, 420.

50 Grünbaum, 1962, 421.

51 Grünbaum 1962, 421.

52 Grünbaum 1962, 421. 
Here we will present the view that, more than epistemologically affine, the conventionality of simultaneity is closely related to Einstein's conventionality of geometry, in the following sense: the conventionality of simultaneity turns out to be one of the conventional elements that makes the chronogeometry conventional. The conventional choice of a particular definition of simultaneity leads to a particular chronogeometry, i.e. the chronogeometry depends on how we define distant simultaneity. ${ }^{53}$

There is a two-fold aspect to the view being presented in what follows: (1) the definition of simultaneity implies a choice of chronogeometry; (2) since it goes hand in hand with the stipulation of the one-way speed of light, it implies the adoption of isotropic electrodynamics or anisotropic electrodynamics, depending on having chosen the standard definition of simultaneity or not. In this way, a non-standard definition of simultaneity results in adopting, instead of the standard isotropic Minkowski spacetime and isotropic electrodynamics $G_{s}+P_{s}$, an anisotropic space-time and anisotropic electrodynamics $G_{n s}+P_{n s}$, in such a way that observationally we have $\mathrm{G}_{\mathrm{s}}+\mathrm{P}_{\mathrm{s}}=\mathrm{G}_{\mathrm{ns}}+\mathrm{P}_{\mathrm{ns}}$.

Let us consider the line element for the anisotropic space-time to understand what is at stake: $d s^{2}=(c d t+q d x)^{2}-d x^{2}-d y^{2}-d z^{2}$, where $q=2 \varepsilon-1 .{ }^{54}$ This line element (and the corresponding non-orthogonal metric tensor ${ }^{55}$ ) results from stipulating that the time at a position $\mathrm{B}$ at $\mathrm{a}$ distance $x$ from the origin $A$ is given by $t_{B}=t_{A}+x / c_{+}$, where $c_{+}=c / 2 \varepsilon$ is the one-way speed of light in the positive $x$ direction. ${ }^{56}$ This stipulation defines the coordinate time, the notion of distant simultaneity, and the one-way speed of light.

We can consider that we still have a Minkowski space-time. ${ }^{57}$ However, it is one which has, as had been noticed by Edwards, ${ }^{58}$ a tilted light cone

$53 \quad$ A related subject is that of the implication of the conventionality of simultaneity thesis in relation to Einstein's practical geometry. This is addressed in Valente, $2017 \mathrm{~b}$.

54 See, e.g., Zhang, 1997, 84; Anderson et al., 1998, 106.

55 See, e.g., Zhang, 1997, 84; Anderson et al., 1998, 111.

56 See, e.g., Zhang, 1997, 8; Jammer, 2006, 180. This expression can be deduced from $\mathrm{t}_{\mathrm{B}}=\mathrm{t}_{\mathrm{A}}+\varepsilon\left(\mathrm{t}_{\mathrm{A}}{ }^{\prime}-\mathrm{t}_{\mathrm{A}}\right)$ by taking into account the expressions for $\mathrm{C}_{-}$and $\mathrm{C}_{+}$.

57 See, e.g., Sonego and Pin, 2009, 26.

58 Edwards, 1963, 489. 
structure due to the anisotropy of the one-way speed of light. This corresponds to an anisotropy of the space-time, even if the local space is Euclidean. This is due to the non-standard definition of coordinate time. We see that the conventional adoption of a particular definition of simultaneity is not extraneous to the chronogeometry of space-time. In fact, a non-standard definition of simultaneity entails a particular metric (or, equivalently, line element) that corresponds to an anisotropic space-time.

The standard isotropic space-time of special relativity incorporates the convention that space is homogeneous and isotropic, corresponding to the assumption of the congruence of distant spatial intervals (having the same coordinate intervals). In fact, in every inertial reference frame the spatial part of space-time is Euclidean. Similarly, it incorporates the assumption of the uniformity of time. When considering a non-standard definition of simultaneity corresponding to an anisotropic one-way speed of light, we maintain these two conventions, but the different convention of distant simultaneity entails a different metric corresponding to an anisotropic Minkowski space-time (i.e. a non-standard chronogeometry).

This case is analogous to that described by Grünbaum that he refers to as an alternative metrization of space: ${ }^{59}$

"Differential geometry allows us to metrize a given physical surface, say an infinite blackboard or some portion of it, in various ways so as to acquire any metric compatible with its topology. Thus, if we have such a space and a net-work of Cartesian coordinates on it, we can just as legitimately metrize the portion above the $x$-axis by means of the metric $d s^{2}=\left(d x^{2}+d y^{2}\right) / y^{2}$, which confers a hyperbolic geometry on that space, as by the Euclidean metric $d s^{2}=d x^{2}+d y^{2} . " 60$

According to Grünbaum, "this metrization commits [us] to regard a segment for which $d x=2$ at $y=2$ as congruent to a segment for which $d x=1$ at $y=1$ ". ${ }^{61}$ This is because "the congruence of two segments is a

59 Grünbaum, 1959, 205-6; Grünbaum, 1962, 414-5.

60 Grünbaum, 1959, 205.

61 Grünbaum, 1959, 205. 
matter of convention, stipulation or definition and not a factual matter". ${ }^{62}$ Equivalently, in our case, by changing the definition of simultaneity the chronogeometry changes. In the case of a standard definition of simultaneity we have $d s^{2}=(c d t)^{2}-d x^{2}-d y^{2}-d z^{2}$, and in the case of a non-standard definition of simultaneity we have $d s^{2}=(c d t+q d x)^{2}-d x^{2}-d y^{2}-d z^{2}$, where $q=2 \varepsilon-1$. However, can we say that the conventionality of simultaneity gives rise to the conventionality of geometry in Einstein's formulation? As we have seen, Einstein's position was not simply that we could change the adopted geometry $(G)$; the position was that by changing the geometrical part of a physical theory, the physical laws $(P)$ could be changed so that no experimental difference arises.

As Edwards, Winnie, and Giannoni's works show, the physical laws indeed change. In particular Giannoni's work shows that when making a definition of simultaneity corresponding to anisotropic one-way speeds of light of $c / 2 \varepsilon$ and $c / 2(1-\varepsilon)$ (in the positive and negative $x$ directions), which entails an anisotropic space-time, electrodynamics gives as a solution that of plane waves propagating in the positive and negative $x$ directions with the conventionally stipulated one-way speeds of $c / 2 \varepsilon$ and $c / 2(1-\varepsilon){ }^{63}$ In this way, when making a non-standard definition of simultaneity we choose a geometry $\left(G_{n s}\right)$ different from the one corresponding to Einstein's standard definition $\left(G_{s}\right)$; and by doing this we also adopt different physical laws, in this case anisotropic relativistic dynamics and anisotropic electrodynamics $\left(P_{n s}\right)$, instead of the standard isotropic relativistic dynamics and isotropic electrodynamics $\left(P_{s}\right)$. However, experimentally $\left(G_{s}\right)+\left(P_{s}\right)=\left(G_{n s}\right)+\left(P_{n s}\right)$, i.e. "only the sum of $(G)+(P)$ is subjected to experimental verification". ${ }^{64}$ We have a case of Einstein's conventionality of geometry. ${ }^{65}$

62 Grünbaum, 1959, 205.

63 Giannoni, 1978, 37-8.

64 Einstein, $1921 \mathrm{a}, 212$.

65 In fact, it is not so much that we can change (P) so that we obtain an observationally equivalent version of the theory; it seems more the case that by changing the definition of simultaneity we change into an anisotropic space-time and we are led to change the physical laws. That is, by changing $(G)$ we do not have the option to choose the remainder of $(P)$ so that there is no contradiction; what happens is that changing $(G)$ has as a consequence a change in $(P):(G)$ and $(P)$ work together as a whole. When we choose a non-standard definition of simultaneity we are also adopting an anisotropic speed of light - the logical 


\section{Conclusions}

In its original formulation, Einstein's conventionality of chronogeometry included two conventional elements: the conventionality of the congruence of spatial intervals (length congruence) and the conventionality of the congruence of successive time intervals (time congruence). The thesis of the conventionality of simultaneity seemed to be an altogether different matter, even if Grünbaum noticed what he called their "complete epistemological affinity". ${ }^{66}$ Here, we made the case that the conventionality of simultaneity as formulated by Reichenbach, Grünbaum, and others, is closely related to Einstein's conventionality of chronogeometry, as another conventional element leading to it. This does not mean that we are proposing the reduction of the conventionality of simultaneity to the conventionality of chronogeometry. What this means is that the conventionality of simultaneity is another element leading to the conventionality of chronogeometry, as the other two conventional elements initially considered by Einstein. The adoption of a particular convention for any one of these three elements leads to a particular chronogeometry $(G)$ and to the adoption of a physical part $(\mathrm{P})$ compatible with this chronogeometry, in a way that the complete structure $(G)+(P)$ is in agreement with experience. That is, the adoption of a particular length congruence, time congruence, and definition of simultaneity - corresponding to a particular chronogeometry - implies the adoption of a specific formulation of the physical laws. For example, in the case of a non-standard definition of simultaneity this leads to the adoption of a non-standard electrodynamics. We can say that, in terms of Einstein's approach, the conventionality of chronogeometry is grounded on: 1) the conventionality of the congruence of spatial intervals; 2 ) the conventionality of the congruence of successive time intervals; 3 ) the conventionality of simultaneity.

circle mentioned by Einstein. This leads to an anisotropic space-time and an anisotropic electrodynamics.

66 Grünbaum, 1962, 421. 


\section{Abbreviations}

[CPAE]: Albert Einstein (1987-). The Collected Papers of Albert Einstein. Ed. by John Stachel et al. 14 Vols., Princeton, Princeton University Press.

\section{References}

Anderson, R., Vetharaniam, I., and Stedman, G.E., 1998, Conventionality of synchronization, gauge dependence and test theories of relativity. Physics Reports 295, 93-180.

Ben-Menahem, Y., 2006, Conventionalism, Cambridge, Cambridge University Press.

Brown, H., 2005, Physical relativity: spacetime structure from a dynamical perspective, Oxford, Oxford University Press.

Darrigol, O., 2015, Mesh and measure in early general relativity. Studies in History and Philosophy of Modern Physics 52, 163-187.

Edwards, W.F.,1963, Special relativity in anisotropic space. American Journal of Physics 31, 482-489.

Einstein, A., 1905, On the electrodynamics of moving bodies. In: CPAE (English translation), Vol. 2, 140-171.

Einstein, A., 1910, The principle of relativity and its consequences in modern physics. In: CPAE (English translation), Vol. 3, 117-142.

Einstein, A., 1911, The theory of relativity. In: CPAE (English translation), Vol. 3, 340-350.

Einstein, A., 1914, The formal foundations of the general theory of relativity. In: CPAE (English translation), Vol. 6, 30-84.

Einstein, A., 1916, The foundation of the general theory of relativity. In: CPAE (English translation), Vol. 6, 147-200.

Einstein, A., 1917, On the special and general theory of relativity. In: CPAE (English translation), Vol. 6, 247-420.

Einstein, A., 1920, Fundamental ideas and methods of the theory of Relativity, presented in their development. In: CPAE (English translation), Vol. 7, 113-150. 
Einstein, A., 1921 a, Geometry and experience. In: CPAE (English translation), Vol. 7, 208-222.

Einstein, A., $1921 \mathrm{~b}$, On a natural addition to the foundation of the general theory of relativity. In: CPAE (English translation), Vol. 7, 224- 228.

Einstein, A., 1922, Four lectures on the theory of relativity, held at Princeton University on May 1921. In: CPAE (English translation), Vol. 7, 261-368.

Einstein, A., 1923, Fundamental ideas and problems of the theory of relativity. In: CPAE (English translation), Vol. 14, 74-81.

Einstein, A., 1924, Review of Albert C. Elsbach, Kant und Einstein. In: CPAE (English translation), Vol. 14, 322- 327.

Einstein, A., 1925, Non-Euclidean geometry and physics. In: CPAE (English translation), Vol. 14, 215-218.

Einstein, A., 1949a, Autobiographical notes. In: P.A. Schilpp (ed.), Albert Einstein: Philosopher-Scientist, 1-94, New York, MJF Books, 1970.

Einstein, A., 1949b, Remarks concerning the essays brought together in this co-operative volume. In: P.A. Schilpp (ed.), Albert Einstein: Philosopher-Scientist, 665-688, New York, MJF Books, 1970.

Giannoni, C., 1978, Relativistic mechanics and electrodynamics without one-way velocity assumptions. Philosophy of Science 45, 17-46.

Giovanelli, M., 2013, Erich Kretschmann as a proto-logical-empiricist: adventures and misadventures of the point-coincidence argument. Studies in History and Philosophy of Modern Physics 44, 11 15-134.

Goenner, H.F.M., 2004, On the history of unified field theories. Living Reviews in Relativity 7, 2.

Grünbaum, A., 1955, Logical and philosophical foundations of the special theory of relativity. American Journal of Physics 23, 450-464.

Grünbaum, A., 1959, Conventionalism in geometry. In: L. Henkin, P. Suppes, and A. Tarski (eds.), Proceedings of the symposium on the axiomatic method, with special reference to geometry and physics. Studies in logic and the foundations of mathematics, Amsterdam, North-Holland Publishing Company. 
Grünbaum, A., 1962, Geometry, chronometry, and empiricism. In: H. Feigl and G. Maxwell (eds.), Scientific explanation, space, and time. Minnesota studies in the philosophy of science, vol. III, Minneapolis, University of Minnesota Press.

Grünbaum, A., 1968, Geometry and chronometry in philosophical perspective, Minneapolis, University of Minnesota Press.

Grünbaum, A., 1976, The duhemian argument. In: S.G. Harding (ed.), Can theories be refuted? essays on the Duhem-Quine thesis, Dordrecht, D. Reidel Publishing Company.

Howard, D., 2010, "Let me briefly indicate why I do not find this standpoint natural": Einstein, general relativity, and the contingent a priori. In: M. Domski, and M. Dickson (eds.), Discourse on a new method: reinvigorating the marriage of history and philosophy of science, La Salle, Open Court.

Jammer, M., 2006, Concepts of simultaneity. From antiquity to Einstein and beyond, Baltimore, The Johns Hopkins University Press.

Janis, A., 2014, Conventionality of simultaneity. Stanford Encyclopedia of Philosophy. https://plato.stanford.edu/entries/spacetime-convensimul/. Accessed 2 February 2017.

Paty, M., 1992, Physical geometry and special relativity: Einstein and Poincaré. In : L. boi, D. flament et J.M. Salanski (eds.), 1830-1930: un siècle de géométrie, de C.F. Gauss et B. Riemann à H. Poincaré et E. Cartan. Epistémologie, histoire et mathématiques, Berlin, Springer.

Reichenbach, H., 1920, The theory of relativity and a priori knowledge, Berkeley, University of California Press, 1965.

Reichenbach, H., 1922, The philosophical significance of the theory of relativity. In: S. Gimbel, A. Walz (eds.), Defending Einstein: Hans Reichenbach's writings on space, time, and motion, Cambridge, Cambridge University Press, 2006.

Reichenbach, H., 1924, Axiomatization of the theory of relativity, Berkeley, University of California Press, 1969.

Reichenbach, H., 1927, The philosophy of space and time, New York, Dover publications, 1957. 
Reichenbach, H., 1928, Space and time: from Kant to Einstein. In: M. Reichenbach, R.S. Cohen (eds.), Hans Reichenbach selected writings, vol 1, Dordrecht, D. Reidel Publishing Company, 1978.

Rynasiewicz, R., 2003, Reichenbach's $\varepsilon$-definition of simultaneity in historical and philosophical perspective. In: F.Stadler (ed.), The Vienna circle and logical empiricism: re-evaluation and future perspectives. Vienna circle institute yearbook [2002] 10, Dordrecht, Kluwer.

Selleri, F., 1996, Noninvariant one-way velocity of light. Foundations of Physics 26, 641-664.

Sonego, S. and Pin, M., 2009, Foundations of anisotropic relativistic mechanics. Journal of Mathematical Physics 50, 042902. E-print 0812.1294 [gr-qc].

Valente, M.B. 2017 a, Einstein's physical chronogeometry. Manuscrito. Revista internacional de Filosofia 40, 241-278.

Valente, M.B. $2017 \mathrm{~b}$, The conventionality of simultaneity in Einstein's practical chrono-geometry. Theoria. An International Journal for Theory, History and Foundations of Science 32, 177-190.

Valente, M.B., 2018, The gauge interpretation of the conventionality of simultaneity. Lato Sensu, Revue de la Société de Philosophie des Sciences (to be published).

Winnie, J.A., 1970, Special relativity without one-way velocity assumptions. Philosophy of science 37, 81-99 and 223-238.

Zhang, Y.Z., 1997, Special relativity and its experimental foundations, Singapore, World Scientific. 